\title{
Not always as simple as a UTI: An atypical case of Lemierre's syndrome, the forgotten disease
}

\author{
Authors: Diviyesh Panchani ${ }^{A}$ and Deepak Nair ${ }^{B}$
}

\begin{abstract}
A 65-year-old man, who was previously fit and well with no known medical comorbidities, was admitted with symptoms of dysuria, urinary frequency, pyrexia, chills and lethargy. His vitals showed that he was hypotensive, tachycardic and pyrexial. Urine dip was heavily positive for leukocytes and nitrites and had markedly elevated inflammatory markers and mild acute kidney injury. He was commenced on piperacillin/tazobactam. He went on to develop severe left-sided neck swelling and erythema. Ultrasound of the neck with subsequent computed tomography revealed extensive internal jugular vein thrombosis with abscess collection around the vein. Microbiology grew Gram-negative bacilli, later identified as Fusobacterium necrophorum. A diagnosis of Lemierre's syndrome was made. The patient was continued on intravenous piperacillin/tazobactam and metronidazole as per sensitivities and later switched to oral metronidazole. He was initiated on warfarin anticoagulation. He made a remarkable recovery and was discharged with outpatient ear, nose and throat and haematology follow-up.
\end{abstract}

KEYWORDS: Lemierre's syndrome, the forgotten disease, Fusobacterium, necrobacillosis, septic thrombophlebitis

DOI: 10.7861/clinmed.2021-0579

\section{Introduction}

Occam's razor states the simplest explanation is usually the most reasonable and correct one. It's a principle that doctors apply daily to make the numerous complex decisions. Infrequently, however, the principle may fail to apply. We present one such case where a simple urinary tract infection (UTI) turned out to be not so simple.

\section{Case presentation}

A 65-year-old man with no significant past medical history presented to the emergency department (ED) with rigors, chills, dysuria, urinary frequency and vomiting for the previous 3 days. Important negative symptoms to note were the absence of shortness of breath, cough and chest pain. In the past, he had suffered from mild gastro-oesophageal

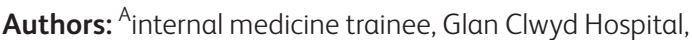
Bodelwyddan, UK; ${ }^{B}$ Clinical fellow, Glan Clwyd Hospital, Bodelwyddan, UK reflux disease, for which he took over the counter antacids, as needed. $\mathrm{He}$ is a retired airline worker, lives with his wife, is fully independent and doesn't smoke or drink alcohol.

His admission vitals showed that he was hypotensive at $83 / 54 \mathrm{mmHg}$, tachycardic at rate of 113 beats/minute, pyretic at

$37.9^{\circ} \mathrm{C}$, oxygen saturation of $98 \%$ on room air and respiratory rate of 19 breaths/minute. On examination, there was no abdominal tenderness, his chest was clear on auscultation, heart sounds were normal and no skin rashes were identified.

Urine dip was positive for nitrites, leukocytes, protein, blood and ketones: haemoglobin $(\mathrm{Hb})$ of $13.4 \mathrm{~g} / \mathrm{dL}$, leukocyte count of $12.5 \times 10^{9} / \mathrm{L}$, neutrophilia of $11.7 \times 10^{9} / \mathrm{L}$, C-reactive protein (CRP) was elevated at $227 \mathrm{mg} / \mathrm{L}$, creatinine of $122 \mu \mathrm{mol} / \mathrm{L}$ (baseline 76).

An initial diagnosis of sepsis (likely from urinary source) was suspected and the patient was started on piperacillin/tazobactam.

\section{Case progression}

Urine microbiology showed no growth but on day 3 of admission, blood culture grew Gram-negative bacilli on two separate anaerobic bottles taken on two separate occasions. Intravenous metronidazole was also added in addition to piperacillin/tazobactam.

On day 4 , the patient developed reduced range of neck movement, painful swallowing and left-sided neck swelling. On examination, he had a firm, erythematous, tender, non-fluctuating and immobile $3 \times 5 \mathrm{~cm}$ neck swelling on the left side.

Ultrasound of the neck revealed a $23 \times 53 \times 48 \mathrm{~mm}$ hypoechoic area at left side of the neck, lateral to the left lobe of thyroid gland and posterior to sternocleidomastoid muscle with low level echoes within, suspicious of abscess formation (Fig 1). Subsequent computed tomography (CT) was performed that confirmed extensive internal jugular vein (IJV) thrombosis and thrombophlebitis (Fig 2).

The Gram-negative bacteria was later characterised as Fusobacterium necrophorum sensitive to metronidazole.

The abscess was drained by ear, nose and throat (ENT) team and the patient was initiated on enoxaparin $1 \mathrm{mg} / \mathrm{kg}$ twice daily which was later switched to warfarin. Following literature review and seeking opinion from different departments, the patient was treated with piperacillin/tazobactam for 10 days and metronidazole for 14 days.

\section{Outcome}

The patient improved clinically and was discharged back home after about a week's stay in hospital with outpatient follow-up in ENT and the haematology department. 


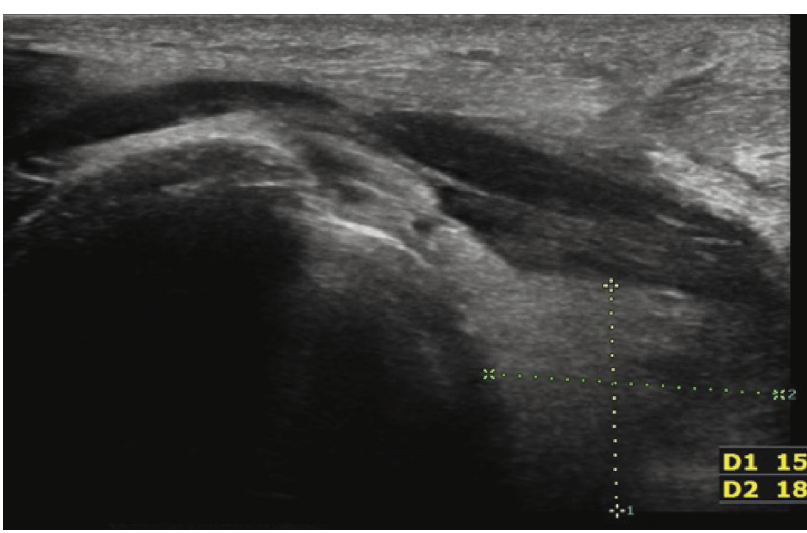

Fig 1. Ultrasound of the neck revealing a $23 \times 53 \times 48 \mathrm{~mm}$ hypoechoic area at left side of the neck, lateral to the left lobe of thyroid gland and posterior to sternocleidomastoid muscle with low level echoes within.

A 2-month follow-up ultrasound showed resolution of abscess and oedema with some residual thrombus and reactive lymph nodes (Fig 3).

\section{Discussion}

Lemierre's syndrome, often referred to as 'the forgotten disease' in medical literature, is a rare but life-threatening condition usually caused by anaerobic bacilli Fusobacterium necrophorum, a normal flora of the oropharynx and paradoxically affects healthy young adults. It was first described by Andre Lemierre, a French bacteriologist, in 1936 by publishing about 20 cases. $^{1}$ The

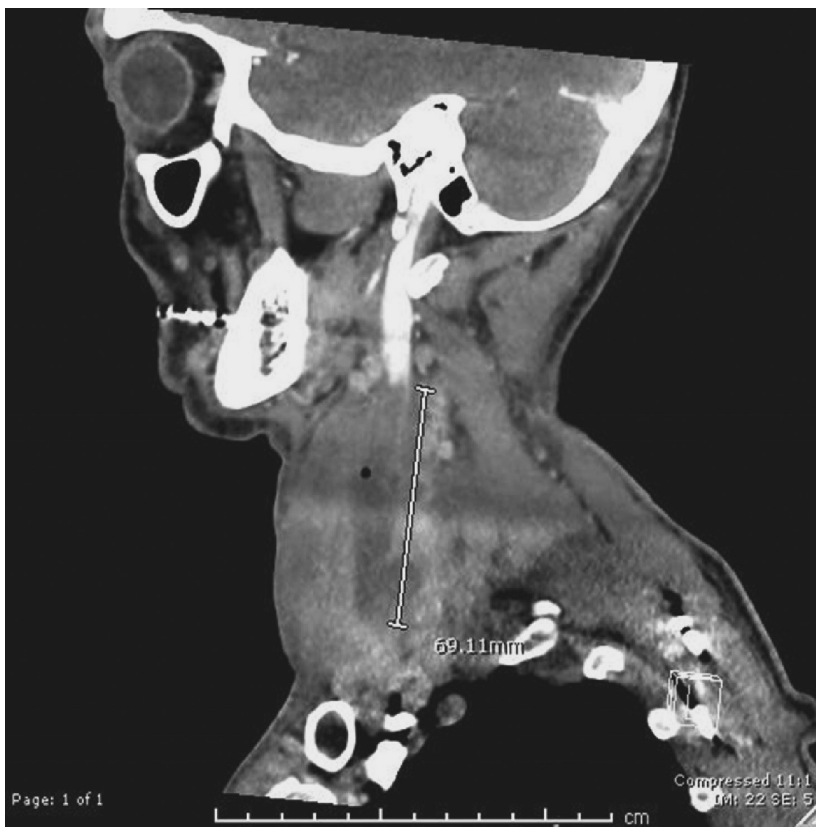

Fig 2. Computed tomography confirming extensive internal jugular vein thrombosis and thrombophlebitis.

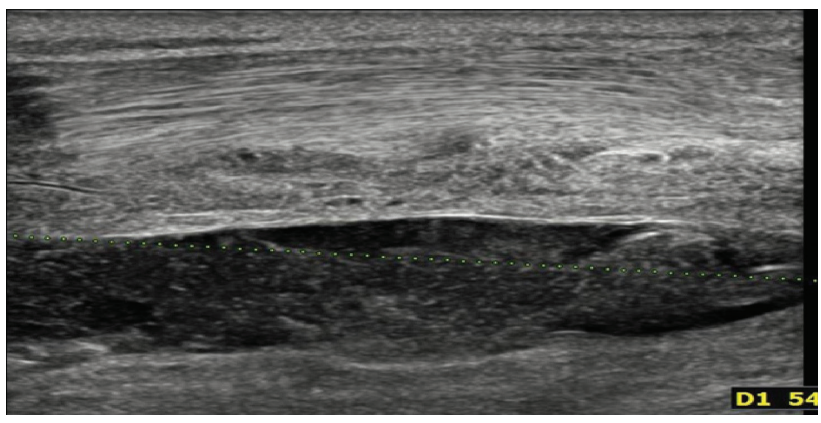

Fig 3. Follow-up ultrasound showing resolution of abscess and oedema with some residual thrombus and reactive lymph nodes.

incidence declined in the late 1940's after the introduction of penicillin for streptococcal infections.

The incidence for the disease is about $1 / 1,000,000 .^{2}$ If not treated early, the fatality, even in the modern era, can be as high as $18 \% .{ }^{3,4}$ It typically affects young and immunocompetent individuals. Most individuals being around at the age of 25 years. ${ }^{4}$ It usually presents with a triad of oropharyngeal infection, septic IJV thrombosis and septic micro-emboli.

The exact pathophysiology of how the bacteria causes septic thrombophlebitis is speculative. Possible explanations are peritonsillar invasion, haematogenous spread from the tonsillar vein or lymphatic spread. 1,4,5

Fusobacterium is generally sensitive to penicillins but a betalactam addition is usually recommended. Empiric antibiotic choices generally include piperacillin/tazobactam, carbapenems, imipenem or meropenem, or ceftriaxone plus metronidazole. ${ }^{4,6}$

Due to the rarity of the disease, data on whether anticoagulation is useful in improving mortality or the best choice of anticoagulant is lacking. Some small studies have suggested the need for anticoagulation only when there is cavernous sinus thrombosis or stroke. For physicians opting for anticoagulation the usual choices remain low-molecular weight heparin or warfarin., ${ }^{1,4}$,

\section{Key points}

> Lemierre's syndrome is a rare but life-threatening disease. Early diagnosis and a multidisciplinary approach are key to appropriate management.

> Urinary symptoms in an otherwise fit and well patient should prompt investigations beyond the genito-urinary system, especially when the patient is not improving as expected.

$>$ Regular clinical examination should follow as new symptoms develop in the management of any patient in clinical practice.

\section{References}

1 Maldonado Sch I, Gutiérrez JMC, Wilkens AR et al. Lemierre's syndrome: An almost forgotten clinical entity. Three case reports and Literature review. Revista chilena de radiología 2015;21:34-40.

2 Sheehan M, McLoughlin D, O'Sullivan R. Fusobacterium necrophorum sepsis after tonsillitis/pharyngitis. BMJ Case Rep 2019;12:e222287

3 Sinave CP, Hardy G], Fardy PW. The Lemierre syndrome: suppurative thrombophlebitis of the internal jugular vein secondary to oropharyngeal infection. Medicine (Baltimore) 1989;68:85-94. 
4 Eilbert W, Singla N. Lemierre's syndrome. Int J Emerg Med 2013;6:40.

5 Tan ZL, Nagaraja TG, Chengappa MM. Fusobacterium necrophorum infections: Virulence factors, pathogenic mechanism and control measures. Vet Res Commun 1996;20:113-40.

6 Golpe R, Marín B, Alonso M. Lemierre's syndrome (necrobacillosis). Postgrad Med J 1999;75:141-4.

7 Stauffer C, Josiah AF, Fortes M, Menaker J, Cole JW. Lemierre syndrome secondary to community-acquired methicillin-resistant
Staphylococcus aureus infection associated with cavernous sinus thromboses. J Emerg Med 2013;44:e177-82.

Address for correspondence: Dr Diviyesh Panchani, Glan Clwyd Hospital, Postgraduate Center, Rhuddlan Road, Bodelwyddan, Rhyl LL18 5UJ, UK.

Email: diviyesh.panchani@doctors.org.uk 